\title{
Determining Parameters for Images Amplification by Pulses Interpolation
}

\section{Determinación de parámetros para amplificación de imágenes mediante interpolación de pulsos}

\author{
Morera-Delfín Leandro \\ Telecommunications' Department \\ Electrical Engineering Faculty \\ Higher Polytechnic Institute Jose Antonio Echeverria \\ E-mail:leandro@electrica.cujae.edu.cu
}

Information on the article: received: June 2013, reevaluated: June, August, and September 2013, accepted: January 2014

\begin{abstract}
This paper presents the implementation of a method for image samples interpolation based on a physical scanning model. It uses the theory to take digital image samples and to perform an implementation of such mechanism through software. This allows us to get the appropriate parameters for the images amplification using a truncated sampler arrangement. The shown process copies the physical model of image acquisition in order to incorporate the required samples for the amplification. This process is useful in the reconstruction of details in low resolution images and for images compression. The proposed method studies the conservation of high frequency in the high resolution plane for the generation of the amplification kernel. A new way of direct application of the physical model for scanning images in analytic mode is presented.
\end{abstract}

\section{Resumen}

Este trabajo muestra la aplicación de un método para la interpolación de muestras sobre una imagen basado en un modelo físico de digitalización de las mismas. Se utiliza la fundamentación teórica de muestreo de la imagen digital y se realiza una implementación de dicho mecanismo a través de un software. Esto permite obtener los parámetros adecuados para amplificar las imágenes utilizando el arreglo de muestreo truncado. Se imita el procedimiento físico de obtención de la imagen y se incorporan las muestras requeridas para la amplificación. Este proceso es útil en la reconstrucción de detalles de imágenes de baja resolución y para la compresión de las mismas. El método propuesto estudia la conservación de las altas frecuencias en el plano de mayor resolución para la generación del núcleo de amplificación y presenta una nueva forma de aplicar directamente el modelo físico de escaneo de la imagen en modo analítico.

\section{Keywords:}

- interpolation

- image reconstruction

- expansion

- sampling

- scanning

\section{Descriptores:}

- interpolación

- reconstrucción de imágenes

- ampliación

- muestreo

- digitalización 


\section{Introduction}

This paper proposes a method for the construction of a pulse interpolation filter with maximum response amplitude at high spatial frequencies. This allows a better reconstruction of the details including the use of the characteristics of this type of amplification in order to achieve linear phase response in the edge transitions of the high resolution image. The preservation of edge information in an accurate frequency range gives a harmonious visual effect to the image and prevents the formation of blocks. This type of filter is used in the amplification, image reconstruction and simultaneously facilitates the compression process.

In some applications, as an imaging amplifier for Space-Multiplexed Optical Transmission (Ozdur et al., 2012) it is necessary to focus/collimate the light beam to the center of the bulk amplifier from the imaging systems (IS 1 and IS 2) of Figure 1 and then to couple back to the output fiber. The amplification effect of the bulk could be seeing as the electrical output of a more dense and sensible photo detector zone integrated over a period of time. During this period the light beam activates the resolution cells, and then the simulation of a photo detector sampling zone is useful to simulate the amplification effects.

The medical-image analysis requires an understanding of sophisticated scanning modalities, constructing geometric models, building meshes to represent domains, and downstreaming biological applications. These four steps form an image-to-mesh pipeline (Levine et al., 2012).

The proposed method could be used as an edgepreserving interpolation method after the denoise process for noisy images. In some cases the image is first decomposed using the bilateral filter into the detail and base layers which represent the small and large scale features, respectively. The detail layer is adaptively smoothed to suppress the noise before interpolation and an edge-preserving interpolation method is applied to both layers, it is effective to employ denoising prior to the interpolation (Jong et al., 2010).

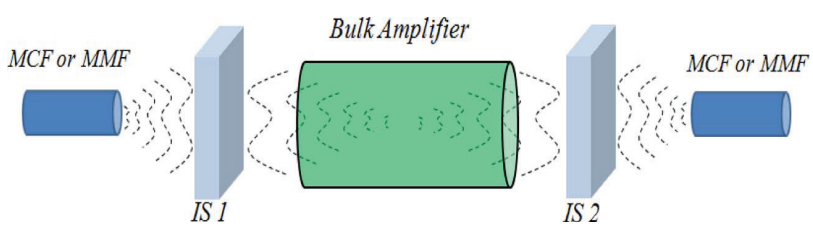

Figure 1. General schematic of the imaging amplifier. (MCF and MMF are the multicore and multimode fibers; IS is the imaging system
Some methods of interpolation, like the curvature interpolation method (CIM), study the edge composition of the low resolution image to interpolate the curvature to the high-resolution image domain. The CIM constructs the high-resolution image by solving a linearized curvature equation, incorporating the interpolated curvature as an explicit driving force (Hakran et al., 2011). Other regression-based image interpolation algorithms have been proposed in the literature, in which the objective functions are optimized by ordinary least squares (OLS). However, it has been shown that interpolation with OLS may have some undesirable properties from a robustness point of view: even small amounts of atypical values can dramatically affect the estimates (Liu et al., 2011).

This investigation tries to find the limits of quality in the high resolution image. The curvature content in the high-resolution image domain is studied using classical theory resources. A restricted interpolation system is made with spatial and window filters in order to increase the high frequency content. The proposed method constructs an interpolation kernel using the high frequency content at high-resolution image domain as an explicit driving force for the variation of the amplification kernel parameters. With this process, the optimal low-high resolution pair is found using the amplification kernel given by the Fourier transform of the truncated sampling arrangement (Papoulis, 1966). In the process filters as Butterworth (Pratt, 2001) and Canny (1986) are used in order to guide the construction of the interpolation kernel and to raise the high frequency content in the high resolution image.

\section{Image sampling system}

In a physical image sampling system the sampling arrangement should be of finite extent. The sampling pulses are of finite width and the image can be sub sampled with spectral overlap. Because of these, spurious spatial frequency components will be introduced into the reconstruction. This effect is called aliasing error (Brown, 1969; Helms and Thomas, 1962) and therefore it is necessary to explore the consequences of the nonideal sampling.

In the example of Figure 2, a thin beam of light goes through a photographic transparency of an ideal image. Passing light is collected in a condenser lens and is sent directly to a photo detector. The electrical output from the photo detector is integrated over a period of time during which the light beam activates a resolution cell. In this type of system is considered that even lens 


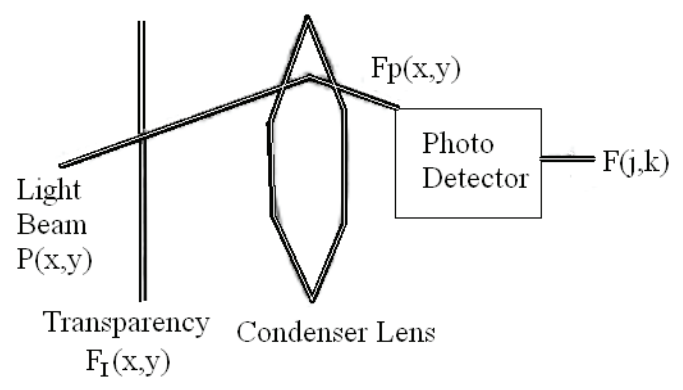

Figure 2. Scheme of the physical model scanning an image, taken from (Pratt, 2001)

with perfect focus produces some blurring because of the diffraction limit of its opening (O'Neill, 1963).

The sampled image is

$F_{p}(x, y)=F_{I}(x, y) \cdot S(x, y)$

where the arrangement of samples is

$$
S(x, y)=\sum_{j=-J}^{J} \sum_{k=-K}^{K} P(x-j \Delta x, y-k \Delta y)
$$

and is comprised of $(2 J+1)(2 K+1)$ identical pulses $P(x, y)$ arranged in a grid spacing $(\mathrm{d} x, \mathrm{~d} y)$. Symmetrical limits of summation are chosen, for notational simplicity, we assume that the sampling points are scaled.

$$
\int_{-\infty}^{\infty} \int_{-\infty}^{\infty} P(x, y) d x d y=1
$$

For purposes of analysis it is assumed that the sampling function is generated by a finite array of Dirac deltas $D_{T}(x, y)$ passing through a linear filter with impulse response $P(x, y)$ then

$$
\begin{aligned}
& S(x, y)=D_{T}(x, y) * P(x, y) \\
& D_{T}(x, y)=\sum_{j=-J}^{J} \sum_{k=-K}^{K} \delta(x-j \Delta x, y-k \Delta y)
\end{aligned}
$$

Taking Equations 1 and 2 yields

$F_{P}(x, y)=\sum_{j=-J}^{J} \sum_{k=-K}^{K} F_{I}(j \Delta x, k \Delta y) P(x-j \Delta x, y-k \Delta y)$

The spectrum of the sampled image is given by

$$
F_{P}\left(w_{x}, w_{y}\right)=\left(\frac{1}{4 \pi^{2}}\right) F\left(w_{x}, w_{y}\right) *\left[D_{T}\left(w_{x}, w_{y}\right) \cdot P\left(w_{x}, w_{y}\right)\right]
$$

where $P\left(w_{x^{\prime}} w_{y}\right)$ is the Fourier transform of $P(x, y)$.

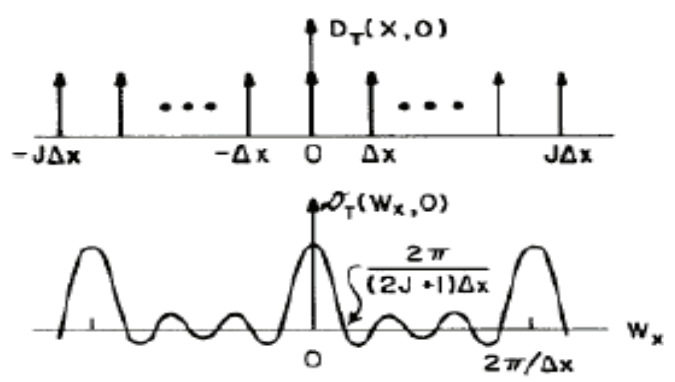

Figure 3. Scheme of the interpolation function, only one coordinate

The Fourier transform of the truncated sampling arrangement (Papoulis, 1966) is

$D_{T}\left(w_{x}, w_{y}\right)=\frac{\sin \left\{w_{x}\left(j+\frac{1}{2}\right) \cdot \Delta x\right\} \cdot \sin \left\{w_{y}\left(k+\frac{1}{2}\right) \cdot \Delta y\right\}}{\sin \left\{w_{x} \cdot \frac{\Delta x}{2}\right\} \cdot \sin \left\{w_{y} \cdot \frac{\Delta y}{2}\right\}}$

This function, evaluated at the limit for large values of $J$ and $K$, is converted into an array of Dirac deltas.

In an image reconstruction system, an image is reconstructed by interpolation of their samples. The interpolation waveforms selected as Sine function or Bessel generally extend over the entire field of the image (Pratt, 2001). If the arrangement is a discontinuous sampling, the reconstructed image will fail near their edges. However, the distance error is negligible in about 8 to 10 samples of Nyquist (Abramatic and Faugeras, 1982).

\section{Modeling the interpolation system}

In modeling the system a test image is taken for amplification by pulses interpolation. The aim is to achieve a new higher resolution image containing the highest fidelity with the original image. The process does not cause pixels block effect and maintains the continuity of phase in the Fourier transform of the result. This allows a smooth visual path. It contains the details in low-frequency areas and facilitates the compression. A convolution kernel (Abramatic and Faugeras, 1978; Pratt et al., 1982; Abramatic and Faugeras, 1982) is generated by the Fourier transform of the truncated sampling arrangement (8).

The space of the band limited functions in the frequency range $\omega \in[-\pi, \pi]$ is spanned by the infinite (yet countable) set of sine functions shifted by integers. Thus any such band limited function $\mathrm{g}(\mathrm{t})$ can be reconstructed from its samples at integer spacing. 
$g(t)=\sum_{n=-\infty}^{n=\infty} g(n) \cdot \sin c(t-n)$

Equations (7) and (9) yield

$$
G_{P}\left(w_{x}, w_{y}\right)=\left(\frac{1}{4 \pi^{2}}\right) G\left(w_{x}, w_{y}\right) *\left[D_{T}\left(w_{x}, w_{y}\right) \cdot P\left(w_{x}, w_{y}\right)\right]
$$

Now consider a spatial linear operator $O\{\cdot\}$ that produces an output image array

$$
Q\left(m_{1}, m_{2}\right)=O\left\{F\left(m_{1}, m_{2}\right)\right\}
$$

The term $O\left\{\delta\left(t_{1}, t_{2}\right\}\right.$ for $t_{i}=m_{i}-n_{i}+1$ is the response, at the output coordinate, to an input of one unit amplitude at coordinate $\left(n_{1}, n_{2}\right)$. It is called the impulse response function array of the linear operator and is written as

$$
\delta\left(m_{1}-n_{1}+1, m_{2}-n_{2}+1 ; m_{1}, m_{2}\right)=O\left\{\delta\left(t_{1}, t_{2}\right)\right\} \text { for } 1 \leq t_{1}, t_{2} \leq L
$$

The impulse response array can change form for each point $\left(m_{1}, m_{2}\right)$ in the processed array $Q\left(m_{1}, m_{2}\right)$. Following this notation, the finite area superposition operation is defined as

$$
Q\left(m_{1}, m_{2}\right)=\sum_{n_{1}=-\infty}^{n_{1}=\infty} \sum_{n_{2}=-\infty}^{n_{2}=\infty} F\left(n_{1}, n_{2}\right) \cdot H\left(m_{1}-n_{1}+1, m_{2}-n_{2}+1 ; m_{1}, m_{2}\right)
$$

This expresses the finite-area superposition operation in the left-justified form in which the input and output arrays are aligned at their upper left corners. It is often notation-wise convenient to utilize a definition in which the output array is centered with respect to the input array. This definition of centered superposition is given by

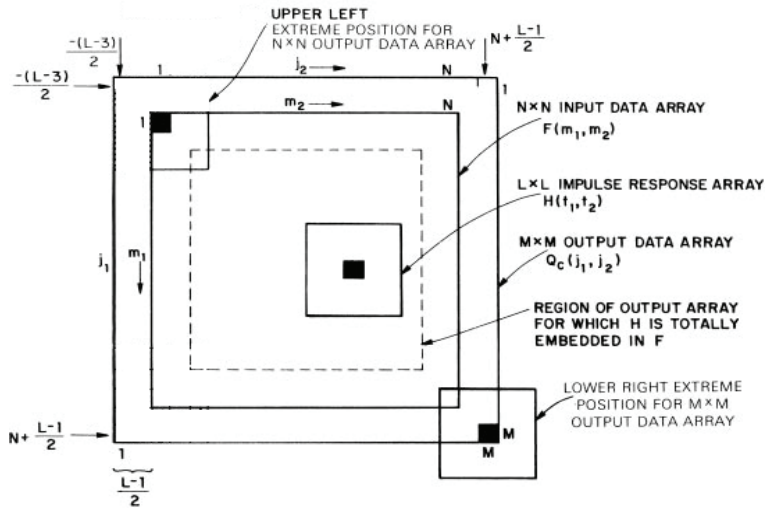

$$
Q_{c}\left(j_{1}, j_{2}\right)=\sum_{n_{1}=-\infty}^{n_{1}=\infty} \sum_{n_{2}=-\infty}^{n_{2}=\infty} F\left(n_{1}, n_{2}\right) \cdot H\left(j_{1}-n_{1}+L_{c}, j_{2}-n_{2}+L_{c} ; j_{1}, j_{2}\right)
$$

The limits of the summation are

$$
-(L-3) / 2 \leq j_{i} \leq L+(N-1) / 2 \text { and } L_{c}=(L+1) / 2
$$

In Figure 4 the examination of the indices of the impulse response array at their extreme positions indicates that $M=N+L-1$, and hence the processed output array $Q$ is of larger dimension than the input array $F$.

In the interpolation system, the input array $\mathrm{F}$ is the low resolution image of $\left(N_{x}, N_{y}\right)$. Then, using $(7),(8)$ and (13), the output data array $Q_{C}\left(j_{1}, j_{2}\right)$ is obtained. Using (10), the samples of $D_{T}\left(\omega_{x}, \omega_{y}\right)$ for the interval in which $\omega \in[-\pi, \pi]$ are inserted. Figure 3 shows how for $\Delta x=1$ and $\Delta y=1, L_{y}=2 \cdot K$ and $L_{x}=2 \cdot J$.

$$
\begin{aligned}
& L_{y}=A_{m p} \cdot K^{\prime}+i n c \cdot c o \\
& L_{x}=A_{m p} \cdot J^{\prime}+i n c \cdot c o
\end{aligned}
$$

where $J^{\prime}$ and $K^{\prime}$ are the dimensions of the low resolution image, this is, the input array F.(24)

$$
\begin{aligned}
& L_{c x}=\left(L_{x}+1\right) / 2 \\
& L_{c y}=\left(L_{y}+1\right) / 2 \\
& Q_{c}\left(j_{1}, j_{2}\right)=\sum_{\omega_{x}=-K^{\prime} / 2}^{\omega_{x}=K_{y}^{\prime} / 2} \sum_{\omega_{y}=J^{\prime} / 2}^{\omega_{y}=J^{\prime} / 2} F\left(\omega_{x}, \omega_{y}\right) \cdot D_{T}\left(j_{1}-\omega_{x}+L_{c x}, j_{2}-\omega_{y}+L_{c y} ; j_{1}, j_{2}\right)
\end{aligned}
$$

Obtaining the impulse response function array in function of the exploration parameters inc and co.

Figure 4. Relationships between input data, output data, and impulse response arrays for finite-area superposition; centered array definition taken from (Pratt, 2001) 


$$
\begin{aligned}
& D_{T}\left(\omega_{x}{ }^{\prime}, \omega_{y}{ }^{\prime} ; j_{1}, j_{2}\right)=\frac{\sin \left\{\left(j_{1}-\omega_{x}+L_{c x}\right) \cdot\left(\frac{L_{x}+1}{2}\right) \cdot \Delta x\right\} \cdot \sin \left\{\left(j_{2}-\omega_{y}+L_{c y}\right) \cdot\left(\frac{L_{y}+1}{2}\right) \cdot \Delta y\right\}}{\sin \left\{\left(j_{1}-\omega_{x}+L_{c x}\right) \cdot \frac{\Delta x}{2}\right\} \cdot \sin \left\{\left(j_{2}-\omega_{y}+L_{c y}\right) \cdot \frac{\Delta y}{2}\right\}} \\
& D_{T}\left(\omega_{x}^{\prime}, \omega_{y}^{\prime} ; j_{1}, j_{2}\right)=\frac{\sin \left\{\left(j_{1}-\omega_{x}+\frac{\left(A_{m p} \cdot K^{\prime}+i n c \cdot c o+1\right)}{2}\right) \cdot\left(\frac{A_{m p} \cdot J^{\prime}+i n c \cdot c o+1}{2}\right) \cdot \Delta x\right\} \cdot \sin \left\{\left(j_{2}-\omega_{y}+\frac{\left(A_{m p} \cdot J^{\prime}+i n c \cdot c o+1\right)}{2}\right) \cdot\left(\frac{A_{m p} \cdot K^{\prime}+i n c \cdot c o+1}{2}\right) \cdot \Delta y\right\}}{\sin \left\{\left(j_{1}-\omega_{x}+\frac{\left(A_{m p} \cdot K^{\prime}+i n c \cdot c o+1\right)}{2}\right) \cdot \frac{\Delta x}{2}\right\} \cdot \sin \left\{\left(j_{2}-\omega_{y}+\frac{\left(A_{m p} \cdot J^{\prime}+i n c \cdot c o+1\right)}{2}\right) \cdot \frac{\Delta y}{2}\right\}}
\end{aligned}
$$

Evaluating the expression for $\omega_{x} \in[-\pi, \pi], \omega_{y} \in[-\pi, \pi]$ and using (19) we obtain the output array $Q_{c}\left(j_{1}, j_{2}\right)$, which has dimensions $\left(M_{x^{\prime}}, M_{y}\right)$, where

$$
M_{x}=N_{x}+L_{x} \quad \text { and } \quad M_{y}=N_{y}+L_{y}
$$

Then the inverse transformation of the output array gives the high resolution image

$$
Q_{S}(x, y)=\sum_{\omega_{x}=-M_{x} / 2 \omega_{y}=-M_{y} / 2}^{\omega_{x}=M_{x} / 2} Q_{c}\left(\omega_{y}, \omega_{y}\right) \cdot \exp \left(j\left(\omega_{x} x+\omega_{y} y\right)\right)
$$

In order to define the parameters inc and co of equations (15) and (16), the high frequency estimate over $Q_{s}(x, y)$ is applied. Applying a Butterworth high pass filter (Pratt, 2001) to less than 5 pixel elements

$$
Q_{S f}\left(\omega_{x i}, \omega_{y i}\right)=\frac{1}{1+\left[\frac{\left(\left(\frac{M_{y}}{10}\right)^{2}+\left(\frac{M_{x}}{10}\right)^{2}\right)^{\frac{1}{2}}}{\left(\omega_{y i}{ }^{2}+\omega_{x i}{ }^{2}\right)^{\frac{1}{2}}}\right]^{2 \cdot}} \cdot Q_{S}\left(\omega_{x i}, \omega_{y i}\right)
$$

Spatial filters for the edge detection over the high resolution image are used in another case. A Canny type filter is applied (Canny, 1986) in order to estimate the amount of edge content and to restrict the parameters $A_{m p}, i n c$ and co of equations (15) and (16). In this case the implementation of the Canny operator gives

$$
Q_{\text {SCanny }}(x, y)=\operatorname{Canny}\left\{Q_{S}(x, y)\right\}
$$

Then the appropriate values of $L_{y}$ and $L_{x}$ can be found in both cases by

$Q_{S f}\left(\omega_{x}, \omega_{y}, L_{x}, L_{y}\right)=Q_{c}\left(j_{1}, j_{2}\right)$

$$
\begin{aligned}
Q_{S o p}\left(L_{x}, L_{y}\right)= & \sum_{x=-M_{x} / 2}^{x=M_{x} / 2} \sum_{y=-M_{y} / 2}^{y=M_{y} / 2} \sum_{\omega_{x}=-M_{x} / 2}^{\omega_{x}=\omega_{y} / 2} \sum_{\omega_{y}=-M_{y} / 2}^{\omega_{y}=M_{y} / 2} Q_{S f}\left(\omega_{x}, \omega_{y}, L_{x}, L_{y}\right) \\
& \cdot \exp \left(-j\left(\omega_{x} x+\omega_{y} y\right)\right)
\end{aligned}
$$

$\operatorname{MAX}\left(Q_{\text {Sop }}\left(L_{x}, L_{y}\right)\right)$

$\left(\frac{\partial^{2} Q_{S o p}\left(L_{x}, L_{y}\right)}{\partial L_{x} \partial L_{y}}\right)=0$

Due to the complexity of the formulation, no analytic solution has been found, but a variational approach has been developed. Defining a fixed value for the large resolution modifier $A_{m p}=1.1$, the maximum responses of high frequencies are found by the output of the filter only for some combination values of the scan parameters inc and co. The high frequencies response of the high resolution image using the Butterworth filter of the equation (20) is shown in Figure 5.

Figures 6 or 8 show how a periodicity exists in each row; for example, if inc $=0.4$ the best result appears every 5 figures and the resolution of the output image increases according to equation (22). For a good result, if inc $=0.4$ then $c o=c o_{0}+n \cdot 5$ when $c o_{0}=4$, equations (15) and (16) get the exact dimensions. But the best result for the Butterworth guide comes from Table 1, $c o=19$, inc = 0.4 where this pattern combination gives us the maxim conservation value of the frequency range of the filter (Figure 9).

$$
\begin{aligned}
& L_{y}=A_{m p} \cdot K^{\prime}+0.4 \cdot\left(c o_{0}+n \cdot 5\right) \\
& L_{x}=A_{m p} \cdot J^{\prime}+0.4 \cdot\left(c o_{0}+n \cdot 5\right)
\end{aligned}
$$

In order to obtain a new vertical resolution, from 182 to 1800 pixels

$$
M_{y}=N_{y}+L_{y}, L_{y}=M_{y}-N_{y}
$$




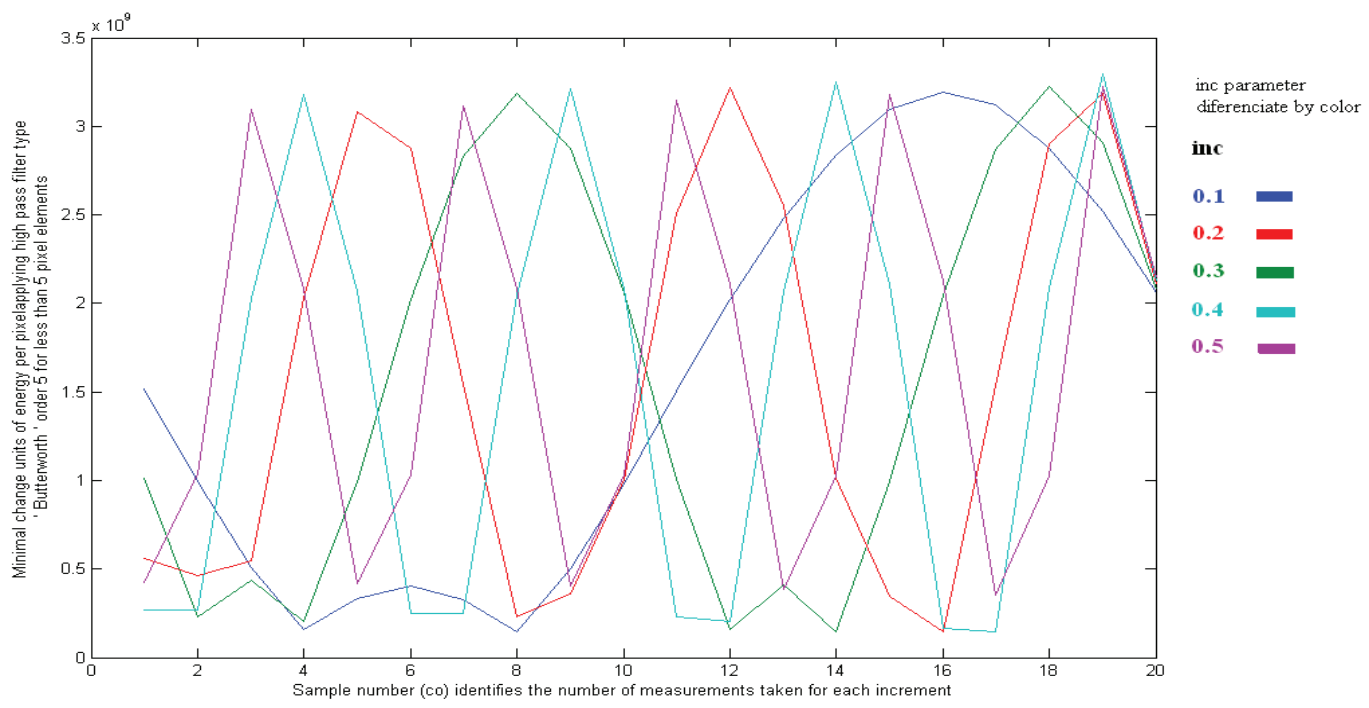

Figure 5. Graphic parameters scan for the output of the filter type 'Butterworth ' (Pratt, 2001), order 5 for less than 5 pixel elements, increment (inc) differentiated per colors and sample number (co) identify the number of measurements taken for each increment

Table 1. High frequency magnitudes after the adjustment process of the amplification kernel. Sum of units of least energy change per pixel result of the high pass filter type Butterworth (Pratt, 2001), order 5 for less than 5 pixel elements

\begin{tabular}{|c|c|c|c|c|c|}
\hline $\mathrm{CO}$ & inc $=0.1$ & inc $=0.2$ & inc $=0.3$ & inc $=0.4$ & inc $=0.5$ \\
\hline 1 & 383928567,687645 & 270095037,508266 & 169198039,912090 & 102292472,923475 & 125127676,957232 \\
\hline 2 & 265336827,105852 & 94633549,2094335 & 129772765,750036 & 90655325,4611820 & 270138137,174281 \\
\hline 3 & 157399882,781851 & 126253386,468731 & 162435251,425276 & 502431645,964924 & 802194399,470288 \\
\hline 4 & 78063421,3240791 & 86932171,0105289 & 502020648,615752 & 829747255,982912 & 514671554,508373 \\
\hline 5 & 105195160,856602 & 265515683,841707 & 798848643,137986 & 513236839,238852 & 123753730,390564 \\
\hline 6 & 115601427,542619 & 501701038,483607 & 733999530,952639 & 99774761,6931317 & 269928660,113030 \\
\hline 7 & 100589995,469657 & 725245838,189243 & 390671166,003094 & 90410729,5035506 & 809589665,431580 \\
\hline 8 & 71483800,9070080 & 827297391,509886 & 95648619,2983738 & 507855576,795838 & 518622467,896560 \\
\hline 9 & 152354026,996521 & 734377407,381142 & 113652216,857252 & 836032023,851749 & 123233632,319536 \\
\hline 10 & 260325092,865537 & 509908457,350087 & 268612928,958653 & 516477468,586911 & 273207463,923044 \\
\hline 11 & 379071451,392629 & 268192909,414039 & 628325957,888577 & 96690369,9074624 & 815764892,457752 \\
\hline 12 & 500332472,602403 & 78953076,5124871 & 836469798,355744 & 88809521,0980179 & 521890543,565737 \\
\hline 13 & 622010395,730627 & 116933760,026838 & 636913647,263801 & 513320418,457530 & 120472385,889034 \\
\hline 14 & 727068317,400424 & 72255954,4438813 & 271311592,423545 & 845105285,844718 & 274630252,046820 \\
\hline 15 & 802300090,381683 & 263321971,361200 & 107661335,427739 & 521099266,043549 & 824783465,459286 \\
\hline 16 & 830828568,075156 & 506052996,661072 & 73031823,6914790 & 80953870,6143567 & 526345829,248504 \\
\hline 17 & 807684329,368176 & 735114700,534598 & 387577748,801602 & 73733511,8314926 & 110166690,342695 \\
\hline 18 & 734377407,381142 & 839728077,836381 & 743205085,474997 & 517243362,064077 & 271945387,044346 \\
\hline 19 & 629973217,428392 & 742412308,021623 & 824984133,789389 & 856772169,300154 & 836016262,233911 \\
\hline 20 & 383928567,687645 & 270095037,508266 & 169198039,912090 & 102292472,923475 & 125127676,957232 \\
\hline
\end{tabular}


$n=\frac{\frac{1618-1.1 \cdot 182}{0.4}-4}{5}, n=708.1$ and $c o=708.1 \bullet 5+4$

Figures 7 and 10 show how a loss of the high frequency content occurs. The application of the interpolation kernel with the Butterworth filter guide produces this effect. This filter does not give the maximum values of frequency of contour information.

The Canny method finds edges by looking for the local maximum of the image gradient. The gradient is calculated using the derivative of a Gaussian filter. The method uses two thresholds to detect strong and weak edges, and includes the weak edges in the output only if they are connected to strong edges. This method is therefore less likely than the others to be deceived by noise, and more likely to detect true weak edges.
$R(x, y)=\frac{\omega_{x s} \cdot \omega_{y s}}{\pi^{2}} \cdot \frac{\sin \left(\omega_{x s} \cdot x\right) \sin \left(\omega_{y s} \cdot y\right)}{\left(\omega_{x s} \cdot x\right) \cdot\left(\omega_{y s} \cdot y\right)}$

The classical separable sine impulse response function array (Pratt, 2001) is

Compare, in Figure 13, the results of the sine separable interpolation (30) and the application of the impulse response function array (20) restricted by the Canny filter over the first column of the high resolution image.

Figure 11 shows the periodicity and the peaks useful for the amplification. The map of figure 12 has localized a zone where exist a maximum value obtained from the Table 2, with $c o=10$ and $i n c=0.5$. Figure 14 shows how the high resolution image maintains the original contrast. This figure has better conserved contour information than the one obtained with the classic interpolation using the impulse response function array (30).

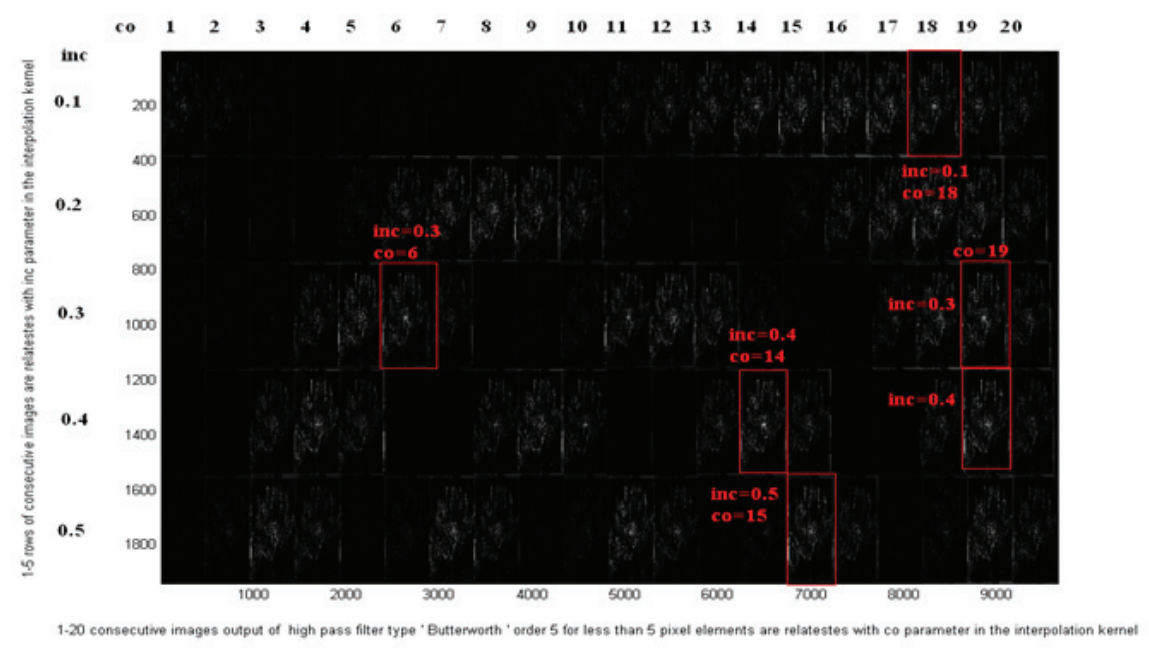

Figure 6. Map of parameter selection for high frequency levels of high pass filter type Butterworth (Pratt, 2001) order 5 for less than 5 pixel elements over the results of the interpolation filter

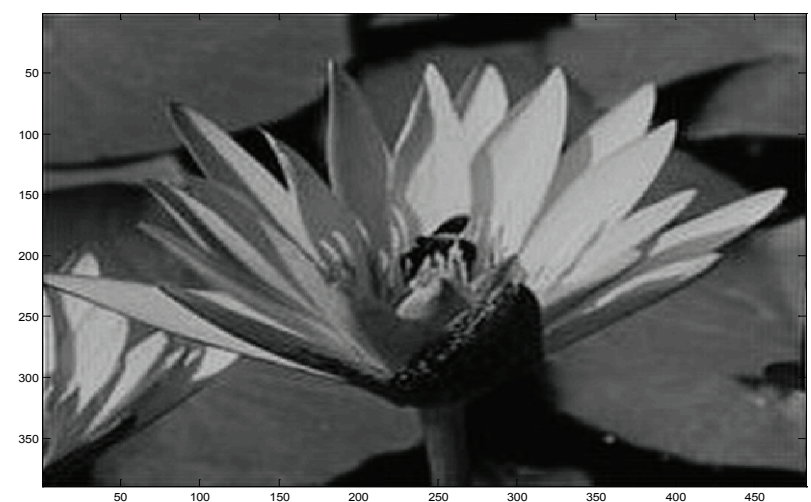

Figure 7. High resolution image whit $\mathrm{Co}=19$ and inc $=0.4$, and the guide of Butterworth (Pratt, 2001), order 5 for less than 5 pixel elements 


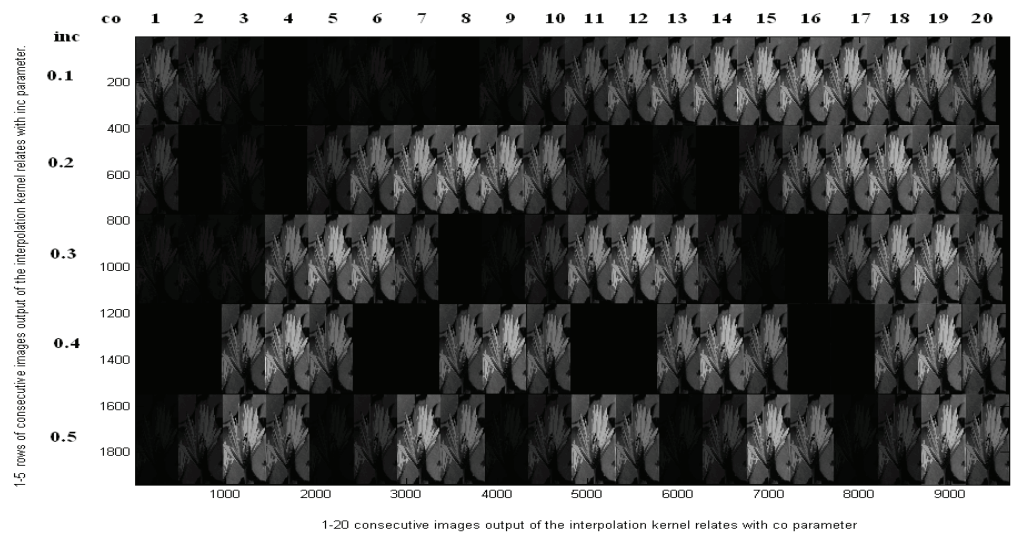

Figure 8. Map of high resolution images obtained by the interpolation kernel varying parameters
In Figure 15, an integer number multiplier is used in order to construct a more dense mesh of the interpolation kernel (20). The parameters in Table 2 for the maximum high frequency response in the high resolution image are used.

In the case of Figure 15, from equation (15)

$L_{x}=13 \cdot\left(1.1 \cdot J^{\prime}+0.5 \cdot 10\right)$

And from equation (16)

$L_{y}=13 \cdot\left(1.1 \cdot K^{\prime}+0.5 \cdot 10\right)$

\section{Discussion and analysis of results}

The investigation shows the main aspects of the interpolation with restriction for the amplification. For instance, which is the goal in the amplification process? What filter could be the guide for the parameters in the construction of the response impulse function or amplification kernel? The Butterworth filter takes in consideration a range of high frequencies, in the case for $\Delta x=5$ pixels, but the main trouble in the interpolation is the contour conservation or high frequencies, that are just over $\pi$. The results of amplification guide by Butterworth give possible combination of parameters for equations (15) and (16) in a range of frequencies. In order to have better results it is necessary to use a filter directly related with the contour information of the image. The edge detectors are developed with a robust capacity for the detection. One famous detector is the Canny filter (Canny, 1986).Then the high frequencies of the interpolation output are related to the impulse response function array using the Canny filter. We found the adequate parameters inc and co in order to make the interpolation kernel, increase the high frequency content in the high resolution image.
Other aspect is the control of the coordinates of amplification of the filter. It is shown in Figures 6 or 8 how periodicities exist in each inc row. The argument of the sine components of the impulse response function array in function (20) are near $\pi / 2$ with the exploration parameters $i n c=0.4$ and $c o=\mathrm{CO}_{0}+n \cdot 5$ for the contour interpolation. The amplification suited under this condition takes the dimensions desired using (15) and (16). Figure 10 is an exact amplification to the precise coordinates. But this process gives losses with the guide of the Butterworth filter. When controlling the amplification it is preferable, for the conservation of high frequency content in the high resolution plane, to find a local maximum using the Canny filter. A whole number is used as multiplier to increase the interpolation kernel dimensions. Another important topic is the comparison applying the classical separable sine impulse response function array (30) and the interpolation results with the impulse response function array (20) constrained by the neighbor detector filter Canny type. It is shown how the restriction increases the range of the pixels values doing a contribution to the high frequency conservation in the high resolution plane. In the last case it is analyzed how the guide of Canny is useful to construct an interpolation kernel in order to obtain a very high resolution image taking only maximum local values in a range. The parameters, when the high frequency content increases, represent the adequate amount of sampler components in the impulse response array $S(x, y)$ of equation (1) for the interpolation. The sampler components are more numerous than the samples of the original image for the interpolation, but the position of these are in the active zone of the resolution cell of the physical scan model and consequently over the valid values of the pixels in the original image and not between. Then the interpolation kernel increases by a whole number multiplier for the adequate parameters $A_{m p}$ inc and co giving a high frequency maximum in an 


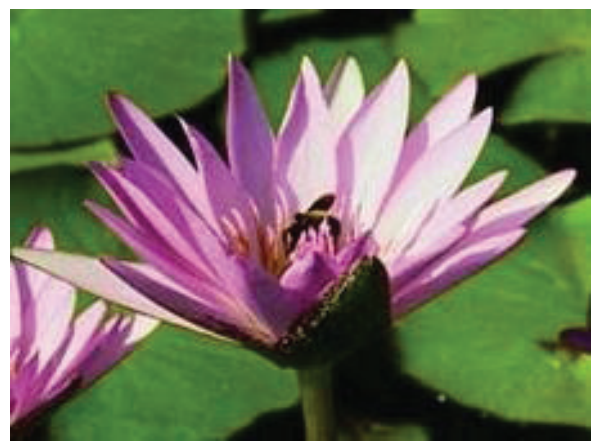

Figure 9. The original test image has $226 \times 182$ pixels

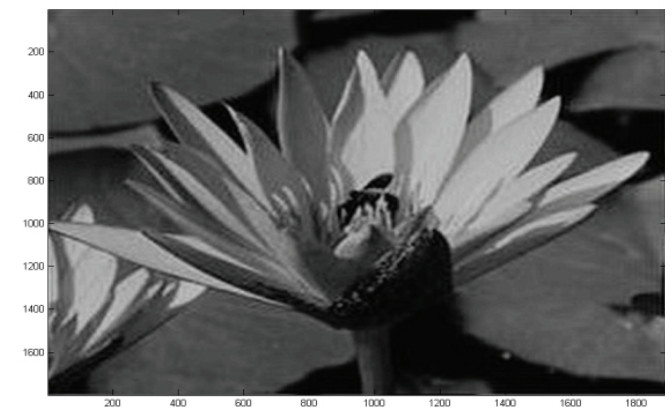

Figure 10. High resolution image using the periodic pattern of Figure 8 for the parameters calculation in a specific amplification from $182 \times 226$ to $1800 \times 2235$ pixels

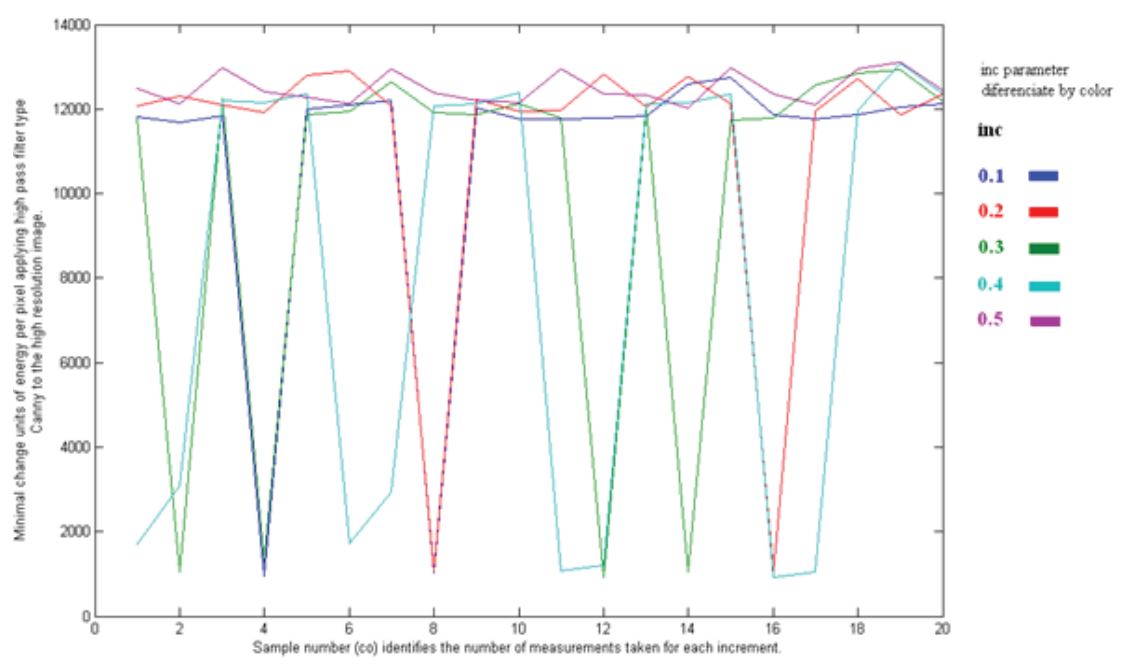

Figure 11. Graphic parameters scan for the output of Canny filter (Canny, 1986) increment (inc) differentiated by colors and sample number (co) identify the number of measurements taken for each increment

Table 2. High frequency magnitudes after the adjustment process of the amplification kernel.

Sum of units of least energy change per pixel resulting from high pass filter type 'Canny' (Canny, 1986)

\begin{tabular}{rrrrrr}
\hline co & inc $=0.1$ & inc $=0.2$ & inc $=0.3$ & inc $=0.4$ & inc $=0.5$ \\
\hline 1 & 12065 & 12636 & 11335 & 1002 & 11687 \\
2 & 12561 & 946 & 13084 & 1382 & 12864 \\
3 & 11091 & 12901 & 11861 & 12466 & 12273 \\
4 & 948 & 1168 & 12206 & 12320 & 12421 \\
5 & 11267 & 12635 & 12138 & 12421 & 11479 \\
6 & 11728 & 12226 & 11924 & 918 & 12766 \\
7 & 11908 & 11766 & 12267 & 1166 & 12255 \\
8 & 1058 & 12127 & 943 & 12445 & 12438 \\
9 & 11784 & 11856 & 12163 & 12323 & 11541 \\
10 & 12414 & 12225 & 12654 & 12416 & 12871 \\
11 & 11984 & 12610 & 12124 & 971 & 12314 \\
12 & 12097 & 930 & 12208 & 1147 & 12457 \\
13 & 11986 & 12871 & 12232 & 12511 & 11578 \\
14 & 11720 & 1057 & 12594 & 12350 & 12824 \\
15 & 12017 & 12603 & 11376 & 12425 & 12402 \\
16 & 12074 & 12130 & 1076 & 925 & 12422 \\
17 & 11949 & 11729 & 12186 & 1063 & 11608 \\
18 & 11856 & 12103 & 11816 & 12252 & 12852 \\
19 & 12171 & 11836 & 12178 & 12354 & 12273 \\
20 & 12225 & 12266 & 12425 & 12422 & 12428 \\
\hline
\end{tabular}




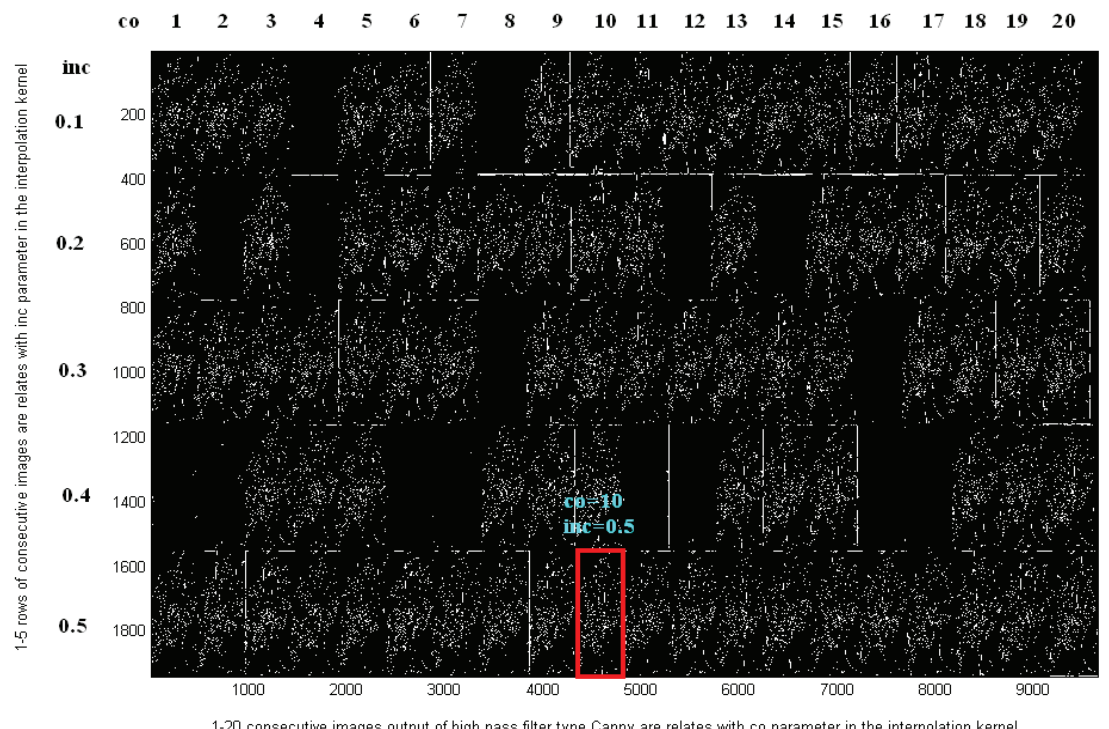

1-20 consecutive images output of high pass filter type Canny are relates with co parameter in the interpolation kernel

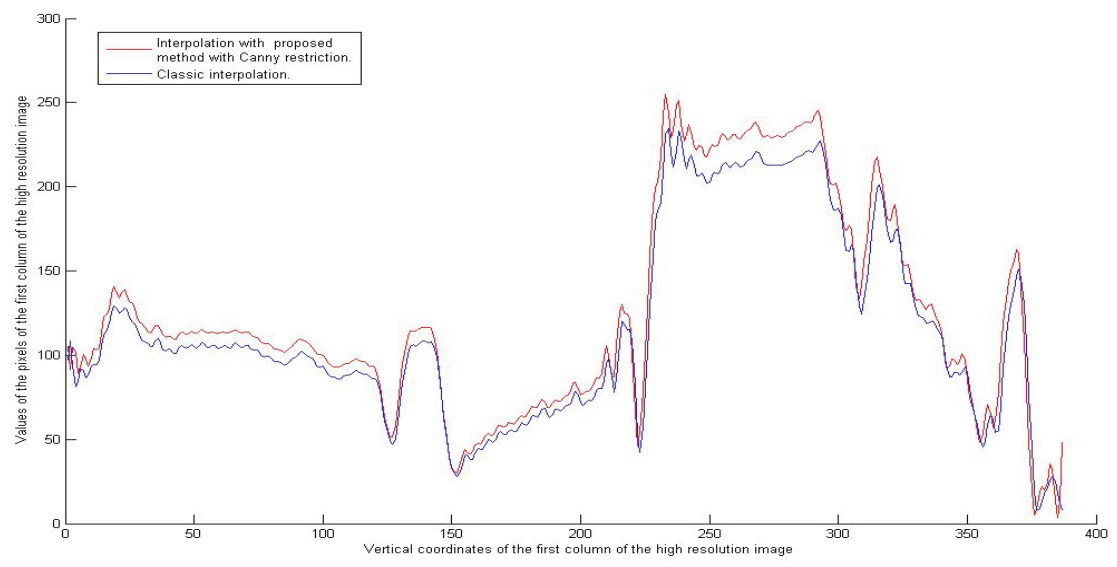

Figure 12. Map of parameters selection for high frequency levels of high pass filter type 'Canny' (Canny, 1986) applied over the results of the interpolation filter
Figure 13. Comparison of the sine separable interpolation (30) and the application of the impulse response function array (20) restricted by the Canny filter over the first column of the high resolution image with $A_{\text {mp }}=1.1$, inc $=0.5$ and $\mathrm{co}=18$

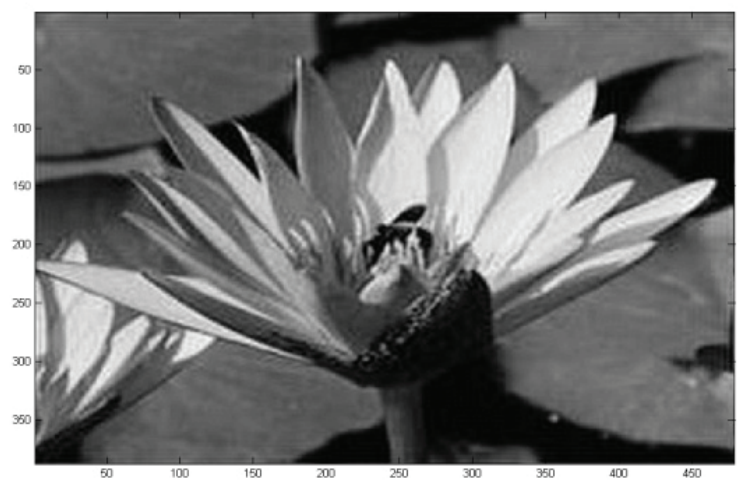

Figure 14. High resolution image for $\mathrm{co}=10$ and $i n c=0.5$, oriented by 'Canny' filter (Canny, 1986) 

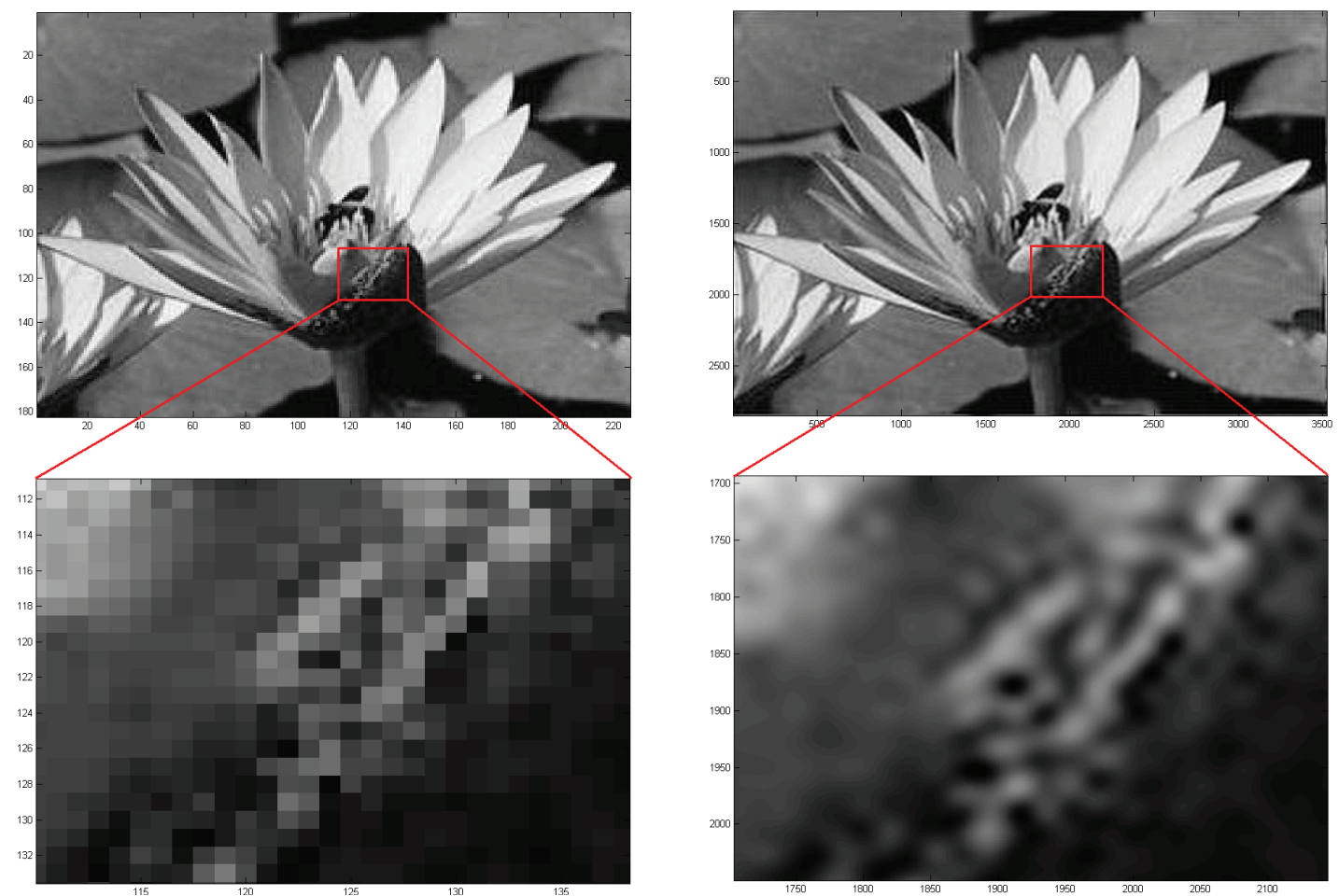

Figure 15. Left side, details of the test image using the interpolation filter, there is a $1550 \%$ amplification of the original image detail from $25 \times 30$ to $387 \times 464$ pixels taken adequate increase step from the right side of equations (15) and (16), original image detail of $25 \times 30$ pixels

initial range. Using this mode we obtained a very high resolution image. The high frequency content in the high resolution plane was retained. Figure 15 shows how this effect occurs over an amplification of the $1550 \%$ in each coordinate.

\section{Conclusions}

This paper shows an effective way for optimizing a pulse interpolation filter in order to obtain the best visual result for the required amplification rate. The proposed amplification method retains the high frequency content and does not produce pixels block effect. A new way of relationship between the constructions of the interpolation kernel and the guide of classic filters is shown in order to increase the high frequency content in the high resolution image. The restriction of the interpolation process using high pass filters increases the range of the pixels values doing a contribution to the high frequency conservation in the high resolution plane. A Canny detector is more effective than the Butterworth high pass filter for the high frequency conservation in the amplification. The characterization of the filter is necessary for the efficient application in different processes of restoration and reconstruction, preserving the characteristics of the primary image. The proposed method makes adequate amplification filters for specific purposes in the field of digital photo restoration and image and video compression.

\section{References}

Abramatic J.F. and Faugeras O.D. Correction to Sequential Convolution Techniques for Image Filtering. IEEE Trans. Acoustics, Speech, and Signal Processing, ASSP-30, 2, April 1982, 346. Simple-Cell Receptive Field Properties by Learning a Sparse Code for Natural Images. Nature, volume 381, 1996: 607-609.

Abramatic J.F. and Faugeras O.D. Sequential Convolution Techniques for Image Filtering, IEEE Trans. Acoustics, Speech, and Signal Processing, ASSP-30, 1, February 1982, pp. 1-10.

Abramatic J.F. and Faugeras O.D. Design of Two-Dimensional FIR Filters from Small Generating Kernels, Proc. IEEE Conference on Pattern Recognition and Image Processing, Chicago, May 1978.

Brown J.L., Jr. Bounds for Truncation Error in Sampling Expansions of Band-Limited Signals. IEEE Trans. Information Theory, IT-15, volume 4 (July issue), 1969: 440-444. 
Canny J. A Computational Approach to Edge Detection, IEEE Trans. Pattern Analysis and Machine Intelligence, PAMI-8, volume 6 (November issue), 1986: 679-698.

Jong-Woo H., Jun-Hyung K., Sung-Hyun C., Jong-Ok K. A Novel Image Interpolation method Using the Bilateral Filter, Consumer Electronics. IEEE Transactions, volume 56 (issue 1), 2010.

Helms H.D. and Thomas J.B. Truncation Error of Sampling Theory Expansions. Proc. IRE, 50, volume 2 (February issue), 1962: 179-184.

Hakran K., Youngjoon C., Seongjai K. Curvature Interpolation Method for Image Zooming, Image Processing. IEEE Transactions, volume 20 (issue: 7), 2011.

Levine J.A, Paulsen R.R., Zhang-Yongjie. Scanning Modalities and Medical-Image Analysis. Computer Graphics and Applications, IEEE, volume 32, 2012.

Liu Xianming, Zhao Debin, Xiong Ruiqin, Siwei Ma. Image Interpolation Via Regularized Local Linear Regression, Image Processing. IEEE Transactions, volume 20 (issue 12), 2011.

O'Neill E.L. Introduction to Statistical Optics, Addison-Wesley, Reading, MA, 1963, pp. 83.

Ozdur-Ibrahim, Shu-Hong, Bass M.,Guifang L. Think Outside the Fiber: Imaging Amplifier for Space-Multiplexed Optical
Transmission. IEEE Photonics Journal Imaging Amplifier for Optical Transmission, volume 4 (issue 5), October 2012.

Papoulis A. Systems and Transforms with Applications in Optics, McGraw-Hill, New York, 1966, p. 105.

Pratt-William K. Digital Image Processing: PIKS Inside, $3^{\text {rd }}$ ed., Copyright (C) 2001, John Wiley \& Sons, Inc. ISBNs: 0-471-37407-5 (Hardback); 0-471-22132-5 (Electronic).

Pratt W.K., Abramatic J.F., Faugeras O.D. Method and Apparatus for Improved Digital Image Processing, U.S. patent 4,330,833, May 18, 1982.

\section{Citation for this article:}

\section{Chicago citation style}

Morera-Delfín, Leandro. Determining Parameters for Images Amplification by Pulses Interpolation. Ingeniería Investigación y Tecnología, XVI, 01 (2015): 71-82.

\section{ISO 690 citation style}

Morera-Delfín L. Determining Parameters for Images Amplification by Pulses Interpolation. Ingeniería Investigación y Tecnología, volume XVI (issue 1), January-March 2015: 71-82.

\section{About the author}

Leandro Morera-Delfin. Graduated in Telecommunications and Electronics Engineering and Master in Biomedical Sciences. He is actually a Professor of Communications Theory and Digital Images and Signals Processing at the Higher Polytechnic Institute Jose Antonio Echeverria, Havana, Cuba. He has job experience in network administration and software development for surveillance and investigations about compressing and pattern recognitions over images and video. 